\title{
Beitrag zur Gliederung des Würms in Mitteleuropa
}

\author{
Von Rudolf Musil und Karel Valoch, Brno \\ Mit einer Tabellen-Tafel
}

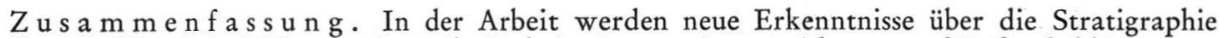
der letzten Eiszeit in Mähren mitgeteilt. Dabei stützen wir uns nicht nur auf Lößaufschlüsse, sondern auch auf zahlreiche Grabungsergebnisse in den Höhlen des Mährischen Karstes, wo es in den Höhleneingängen gelegentlich zu Verknüpfungen der typischen Lößbildungen mit den viele paläontologische und archäologische Funde enthaltenden Höhlensedimenten gekommen ist. Wenn wir daher rein stratigraphische Erkenntnisse auswerten, stützen wir uns doch in großem Maße auf die eingeschlossenen Funde.

Wir kommen zu dem Schlusse, daß die Würmeiszeit eine stark gegliederte Periode darstellt. Aus diesem Grunde geben wir die von uns bisher benützte Bezeichnung der Interstadiale durch Abkürzungen auf und ziehen die Benennung nach den typischen Lokalitäten vor. Wir bringen weitere Beweise für die Existenz des Mittelwürm-Interstadials, welches wir mit dem Namen Interstadial Podhradem bezeichnen.

$\mathrm{Sum}$ mary. The paper evaluates new information bearing upon the stratigraphy of the last Ice Age in Moravia. In reaching their conclusions not only the loess outcrops are considered but also the results of numerous investigations into the caves of the Moravin Karst region. Here, in cave entrances, typical loess and cave sediments are occasionally found together. They contain many palaeontological and archaeological remains.

As it is concluded that the Würm period is one of many component parts the generally current interstadial terms are not used, instead names are derived from typical localities of the area. Additional evidence is cited for the existence of a Middle Würm interstadial to which the name Pohradem Interstadial is given.

\section{Vergleichende Beobachtungen an Profilen und Funden}

Fragen der Gliederung der letzten Eiszeit stehen im Vordergrund eines regen Interesses von mehreren Quartärdisziplinen. Dies ist begreiflich, da die stratigraphischen Unterlagen für eine ganze Reihe verschiedener Fachwissenschaften wichtig sind. Bei der Voraussetzung einer Dreiteilung des Würms hat man die letzte fossile Schwarzerde, die manchmal verdoppelt war, als den aus dem ersten Interstadial stammenden Boden betrachtet. Die Bestimmung dieses Bodens stützte sich hauptsächlich auf letztinterglaziale paläontologische Funde in tieferliegenden Böden (zusammenfassend V. LožEK 1955), auf den Vergleich mit ähnlich gelagerten Böden in anderen Lößprofilen und im böhmischen Raum auf Beziehungen zu Flußterrassen. Diese als Würm 1/2 bezeichneten Böden wurden dem Boden von Göttweig zeitlich gleichgesetzt.

In unserer Arbeit wollen wir uns hauptsächlich auf Lößprofile aus dem Raume von Mähren und auf Grabungen in den Höhlen des Mährischen Karstes stützen. Aus allen Arbeiten (J. Pelíšek, K. Žebera, V. Ložek, J. Kukla, H. Seichterová, J. Demek, T. Czudek, B. Klíma, R. Musil, K. Valoch) geht hervor, daß sich die Schichtenfolge ständig wiederholt, daß einzelne Schichten auf diesem kleinen Raum immer gleiche makroskopische Eigenschaften aufweisen. Wenn man mit dem Holozän beginnt, kann man folgendes Schema wiedergeben.

Unter der holozänen Schwarzerde liegt der jüngste Löß (W 3) an vielen Stellen mit jungpaläolithischen und damit verknüpften paläontologischen Funden (Magdalénien: die Höhlen Kůlna, Švédův stůl, Adlerova; Gravettien: Pavlov 24.800 150 B.P.). Im Liegenden dieses Lösses befindet sich eine braune, schwach verlehmte Schicht (W 2/3), gleichfalls mit zahlreichen Funden (Höhle Pod hradem 26.240 2300 B. P., Dolní Věstonice $25.600 \pm 170$ B.P.). Der darunterfolgende Löß (W 2) weist fast immer starke Solifluktions- 
einwirkungen auf; paläontologische Funde sind meist nur aus Höhlen und ihren Eingängen bekannt. Diese Schichten, deren Zeitbestimmung von allen Forschern übereinstimmend durchgeführt wird, liegen immer auf einem schwarzen humosen Boden, im Löß noch mit einer rostbraunen Schicht, die als B-Horizont bezeichnet wurde (W 1/2). In den Höhlen enthält dieser Boden paläolithische und häufige paläontologische Funde. Der Löß im Liegenden (W 1), in Mittelmähren der mächtigste von den hier beschriebenen, bedeckt einen meist mächtigen Bodenkomplex des letzten Interglazials.

Die als W $1 / 2$ bezeichneten Böden unterscheiden sich durch ihr Aussehen sehr schwer von interglazialen Böden, sind aber grundsätzlich anders als die schwach verlehmte Schicht der W 2/3-Schwankung. Es ist der letzte intensive fossile Boden. In letzter Zeit haben manche Forscher angenommen, daß sein B-Horizont ein selbständiger Boden von Lessivé-Typus ist, der ein interglaziales Klima kennzeichnet. Auf Grund dieser Feststellung wurden dann diese Böden dem letzten Interglazial zugewiesen. Die Lessivation der Böden muß man als einen pedologisch vor sich gehenden Prozeß auffassen, was bedeutet, $\mathrm{da} ß$ man ihn, abhängig von äußeren Bedingungen, in verschiedenen Entwicklungsstadien erfassen kann. Daraus geht hervor, daß die Intensität der Lessivation jedenfalls mit Hilfe der Laboratoriumsanalysen durchgeführt werden sollte, um über die zu ihrer Entstehung nötigen klimatischen Ansprüche entscheiden zu können.

Im Verlaufe der Tagung der tschechoslowakischen Geologischen Gesellschaft in Brno 1963 führte auch eine Exkursion in den Aufschluß der Ziegelei in Modřice. Auf der heutigen, in ungünstigem Beobachtungszustande sich befindenden Abbauwand sah man unterhalb des Holozäns eine mächtige, durch undeutliche braune Zonen getrennte Lößschicht, die einem verdoppelten Bodenkomplex auflag. Im Liegenden der unteren Humuszone befand sich ein eindrucksvoller rostbrauner Horizont, den die anwesenden Teilnehmer der vorher hier stattgefundenen Exkursion der INQUA-Subkommission für Lößstratigraphie als typischen Lessivé bezeichnet hatten. Die Teilnehmer der genannten Subkommission hatten sich geeinigt, daß dieser Bodenkomplex dem R/W-Interglazial einschließlich der Frühwürm-Interstadiale (Amersfoort, Brörup) entspräche, so wie das ganze Profil die typische Entwicklung des Würms zeige (FInk 1963). Dieser Komplex wurde von V. LožEK als PK II+III, die oberen braunen Zonen als PK I bezeichnet (LožEK 1964).

Schon im Jahre 1940 und wiederholt 1949 spricht J. PeLíšEK bei der Beschreibung dieser Ziegelei von zwei durch einen mächtigen Löß getrennten Bodenschichten. Unterhalb des voll ausgebildeten W 2/3 und W 2 lag nach PELíšEK eine degradierte Schwarzerde mit einem etwa $50 \mathrm{~cm}$ dicken rostbraunen B-Horizont (W 1/2); tiefer folgte ein mehr als $4 \mathrm{~m}$

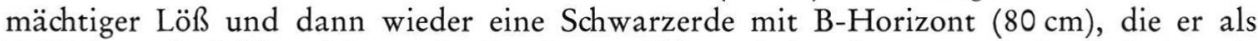
R/W-Interglazial bezeichnete. Im Laufe der Abbauarbeiten konnten wir beobachten, wie es an der Profilwand allmählich zu gewissen Veränderungen kommt. Bei unserer Bearbeitung dieses Aufschlusses im Jahre 1953 konnten wir schon diese Schichtfolge feststellen (Musil, Valoch, NečEsaný 1955 (118): Unterhalb des Holozäns lag der jüngste Löß mit Pseudomyzel und einzelnen Konkretionen (W 3), in seinem Liegenden eine schwache braune lössige Schicht (W 2/3). Es folgte ein lichter Löß (W 2) mit Pseudomyzel, stellenweise durch Solifluktion geschichtet. Die W 1/2-Schicht, die vor wenigen Jahren (vgl. PELíšEK 1949) voll ausgeprägt sichtbar gewesen war, war nunmehr als ein brauner, stellenweise Eiskeile mit dunkler Ausfüllung enthaltender Lehm zu beobachten. Der tieferliegende Löß (W 1) erreichte schon nicht jene Mächtigkeit, die noch PELíšEK angetroffen hatte, und bedeckte zwei Humuszonen, eine jede mit einer eigenen rostbraunen Schicht, die wir damals als B-Horizont bezeichnet haben (R/W). Aus der unteren Humuszone liefen Eiskeile aus. Die beiden Bodenkomplexe lagen damals unmittelbar aufeinander. Das Profil war unten mit einem mächtigen Lößkomplex $(R)$ abgeschlossen. Im Jahre 1953 waren also noch alle durch PELíšEK beschriebenen Schichten anzutreffen, obgleich in abweichender Entwicklung. Der untere Bodenkomplex ist mit jenem Lessivé-Horizont, den 
die Teilnehmer der INQUA-Subkommission 1963 als letztinterglazial bezeichnet hatten, identisch.

Bei der Gelegenheit des VI. INQUA-Kongresses in Polen 1961 haben J. KukLA und V. LožEK ein Profil von Modřice abgebildet, welches ungefähr dem heutigen Stand entspricht. Der ursprünglich ausgeprägte Boden mit rostbraunem Unterboden (unser W 1/2) fehlt jetzt schon und ist in dem von den Verfassern als "Paudorf" bezeichneten Komplex verborgen. Der liegende, dem W 2 zugesprochene Löß befindet sich auf einem Komplex humoser Böden mit zwei unkomplett ausgebildeten Lessivé-Horizonten, von denen der obere als „Göttweig" (offenbar im alten Sinne des Wortes als W 1/2) und der untere als „Eem + W 1 “ eingereiht wird.

Der beschriebene Aufschluß von Modřice, dessen Ablagerungen ohne exakte Grundlagen, je nachdem, was zu einer gewissen Zeit zufällig sichtbar war, verschiedentlich stratigraphisch eingereiht wurde, stellt in seiner Vollentwicklung ein typisches Würm-Profil in der Umgebung von Brno vor.

Heutzutage ist das besterhaltene Profil durch jungpleistozäne Ablagerungen in der Ziegelei Židenice I (Malá Klajdovka) zu sehen, welches wir bereits 1955 beschrieben haben und seither ähnlich wie die übrigen hiesigen Lößaufschlüsse ständig verfolgen. Unter dem Holozänboden befindet sich der jüngste Löß $(120 \mathrm{~cm}$; W 3) mit einer blaßbraunen Zone im Liegenden (W 2/3). Dann folgt ein stellenweise durch Solifluktion geschichteter Löß (W 2), der einem dunklen, humosen, horizontal verlaufenden Boden mit stark entwickeltem B-Horizont (W 1/2) aufliegt. Der Erhaltungszustand dieses Bodens ist verschieden; stellenweise ist davon nur der rostbraune B-Horizont übriggeblieben, stellenweise ist er vollkommen abgetragen. Diese beiden Bodenschichten entsprechen den beiden, heute als schwache Lehmschichten entwickelten Böden in Modřice. Im Liegenden befindet sich eine bis $6 \mathrm{~m}$ mächtige Lößschicht (W 1), die einen Bodenkomplex bedeckt. Dieser Bodenkomplex, genau in unseren früheren Arbeiten beschrieben, besteht aus dunklen, immer mit rostbraunen Verlehmungsschichten verknüpften Humuszonen, die etwa im oberen Drittel durch eine bis $70 \mathrm{~cm}$ mächtige Lößschicht getrennt sind. Diesen ganzen Komplex haben wir früher als letztinterglazial und die trennende Lößschicht als Präwürm bezeichnet. Auf Grund der neuen Erkenntnisse betrachten wir als interglazial nur den unteren mächtigeren Teil dieses Komplexes, die Lößeinlage als die erste klimatische Oszillation des beginnenden Würmglazials. Dem entsprechen auch die durch Löß ausgefüllten, bis $40 \mathrm{~cm}$ langen Keile, die in den unteren Bodenkomplex eindringen (vgl. l. c. 1955, Profil G). Diese Entwicklung der beschriebenen Schichten entspricht dem heutigen Stand in Modřice, wo durch die erwähnte Kommission der untere Teil als Eem + Amersfoort, der obere als Brörup bezeichnet worden ist. Auch die diesem Bodenkomplex aufliegenden Schichten in Židenice I und Modřice sind völlig gleich ausgebildet.

Bei den im Jahre 1964 durch J. Sýков in der Ziegelei Židenice I durchgeführten Arbeiten gelang es, das bisher sichtbare Profil durch die Abfolge der liegenden Schichten zu ergänzen. Unter dem Bodenkomplex befindet sich ein gelbbrauner Löß $(200 \mathrm{~cm})$, der allmählich in einen dunkelbraunen Boden $(70 \mathrm{~cm})$ übergeht, welcher einer braunen, würfelig zerfallenden Erde mit $\mathrm{CaCO}_{3}$-Pseudomyzel $(50 \mathrm{~cm})$ aufliegt. Darunter folgt ein entkalkter Löß mit Feinsandbeimischung, oben zuerst von brauner Farbe. Nach unten zu wird der Löß heller und toniger (insgesamt $270 \mathrm{~cm}$ ). An der Basis befindet sich eine Flußterrasse, die der Terrassenstufe von Turany entspricht.

Die angeführten Aufschlüsse zeigen uns die vollgegliederte Abfolge des Jungpleistozäns, wie wir diese bereits 1955 festgelegt haben. Ein Unterschied besteht nur in der Beurteilung des Bodenkomplexes. Im Unterschied zu dem Interpretationsversuch in Modŕice vermuten wir, daß dem Eem der ganze untere Teil des Komplexes angehört, dem Amersfoort dagegen der ganze obere Teil. Dazu führt uns die Tatsache, daß in den Auf- 
schlüssen von Židenice I und Modřice im unteren Teil des mächtigen Lösses (W 1), etwa $90 \mathrm{~cm}$ über dem ganzen Bodenkomplex, noch eine ungefähr $50 \mathrm{~cm}$ starke braune Lehmschicht liegt. Diesen Boden, den wir im Verlaufe der Abbauarbeiten schon vor Jahren beobachten konnten, möchten wir heute dem Brörup gleichsetzen. Die chronologische Einstufung der beschriebenen Schichten wurde nur auf Grund der Stratigraphie durchgeführt. Dabei stützt sie sich auch auf die volle Übereinstimmung mit dem Profil bei Amersfoort (ZAGWIJN 1961).

An die Brünner Umgebung mit mächtigen und reichgegliederten Lößanwehungen knüpft unmittelbar der $\mathrm{Mährische} \mathrm{K}$ arst an, in dessen Höhlen durch zahlreiche Grabungen die Stratigraphie der Ablagerungen erschlossen wurde. Im südlichen Teil des Karstes wurde durch das Archäologische Institut der Akademie die wenig tiefe, breit geöffnete Höhle Švédův stůl gegraben mit folgender Schichtfolge (Beschreibung nach B. KLíma 1962): Unter dem Holozän befand sich ein Löß mit viel Kalksteinschutt im unteren Teil (W 3). Tiefer folgte eine lössige braune Schicht (W 2/3), die wieder auf einem Löß (W 2) lag. Im Liegenden dieser Schichten befand sich ein mächtiger, gegliederter Braunerdekomplex (W 1/2 $+\mathrm{R} / \mathrm{W}$ ) mit ockerfarbigem, tonigem Höhlenlehm an der Basis, dem Felsboden aufliegend.

Im nördlichen Karst haben wir die Höhle Pod hradem gegraben, in der wir vom Eingange bis $30 \mathrm{~m}$ tief ins Höhleninnere diese Stratigraphie verfolgen konnten: Unter dem Holozän lag ein im Eingange in zwei Schichten gegliederter Löß (W 3), in dessen Liegenden sich eine blaßbraune Schicht mit viel Steinschutt (W 2/3) befand. Dann folgte wieder eine Lößschicht (W 2) und darunter ein etwa $3 \mathrm{~m}$ mächtiger Schichtkomplex von humosen Böden (W 1/2). Nach unten zu war dieses Profil wieder durch einen Löß abgeschlossen (W 1).

Die Datierung dieser beschriebenen Profile erfolgte nicht nur auf Grund der Stratigraphie, sondern auch mit Hilfe der paläontologischen und archäologischen Funde. Die angeführte Beschreibung zeigt, daß die Schichtenfolge der Höhlensedimente und der Lösse im freien Lande dieselbe ist.

Die meisten Vertebratenfunde stammen aus Höhlen; doch werden bei archäologischen Grabungen der Freilandsiedlungen auch Mengen derartigen Materials gewonnen. Vergleiche der Faunengemeinschaften zeigen, daß es möglich ist, einzelne Schichten nicht nur zwischen den Höhlen untereinander, sondern auch mit den Freilandaufschlüssen zu parallelisieren. Es zeigte sich, daß eine markante Scheide das Ende der Bildung der braunen humosen Böden, die wir als Würm $1 / 2$ bezeichneten, vorstellt. Von großer Bedeutung ist, daß diese interstadiale Faunenassoziation auf einer kalten stadialen Gemeinschaft des Frühwürms liegt (Musıl 1965). Nach dieser Zeit ändert sich die ganze Gemeinschaft, es verschwinden einige Arten, die entweder bis zum Ende des Pleistozäns überhaupt nicht mehr erscheinen oder deren Vorkommen in dieser Zeit sich stark vermindert. Das folgende Stadial ist durch den plötzlichen Einbruch und starkes Anwachsen der kälteliebenden Arten gekennzeichnet. Diese auffällige Grenze kann man bei genügender Fundanzahl immer beobachten, und man kann vermuten, daß sie nicht nur in unserem Gebiet feststellbar sein wird. Wasser- und wärmeliebende Tiere verschwinden vollkommen. Dabei handelt es sich nicht bloß um eine Temperaturverminderung, sondern um tiefgreifende Änderungen des ganzen Lebensmilieus. Die für die vorangehende Periode charakteristischen großen Mengen von Auerochsen und Wisenten und gleichfalls die großen, dem Maral ähnlichen Hirsche erscheinen jetzt nur noch sporadisch und zwar bis am Ende des Pleistozäns. Auch in der Gesamtzahl der Höhlenbären äußert sich diese Grenze markant.

Die Veränderungen in einzelnen Zeitabschnitten betreffen nicht nur ganze Gemeinschaften; man kann dies auch bei einzelnen Arten beobachten, wie z. B. im Bau der Zähne 
der Höhlenbären, der Pferde usw. In den Schichten des W 1/2 findet man Pferdezähne noch mit relativ primitiven Kennzeichen, die man in zwei Gruppen aufteilen kann: In der ersten Gruppe sind schwer gebaute Pferde der Equus mosbachensis-abeli-Gruppe, in der zweiten Equus germanicus. Während die Art Equus germanicus im W 2 weiterlebt, bedeutet das Sedimentationsende des W 1/2 auch das letzte Erscheinen der Gruppe der schweren Pferde. Dieser Zeitpunkt bildet somit eine bedeutsame Grenze. Eine ähnliche Grenze in der Ausbildung der Pferdezähne kann man auch zwischen dem W 2 und W 3 beobachten. Auch bei den Höhlenbären kann man klimatisch bedingte Veränderungen an den Zähnen feststellen (Musil 1965).

Es ist somit möglich, auf Grund der ganzen Faunengemeinschaften wie auch auf Grund der Veränderungen einzelner Arten und der wechselnden individuellen Anzahl bei genügender Menge des paläontologischen Materials eine entsprechende stratigraphische Einreihung durchzuführen.

Einen Anhaltspunkt für die Gliederung bieten auch die malakozoolog is $\mathrm{ch}$ e $\mathrm{n}$ $\mathrm{Funde}$. Die Malakofauna des letzten Interglazials unterscheidet sich auffallend von den folgenden Interstadialen, wie LožEK in seinen Arbeiten betont. Im Lößaufschluß von Litoměrice (Leitmeritz) konnte er auf Grund dieser Funde feststellen, daß sich der obere Boden, den er damals in Hinsicht auf seine stratigraphische Position als das Göttweiger Interstadial im Sinne von H. Gross (LožEK, KuKLA 1959, 98) bezeichnete, klimatisch vom Interglazial scharf unterscheidet und deshalb als Interstadial gewertet werden muß. Wichtig ist, daß „... der Versuch, die jungpleistozänen Warmphasen zu charakterisieren, ... zur Feststellung scharfer Unterschiede zwischen dem letzten Interglazial und dem Göttweiger Interstadial ..." führte (1. c., 103). In stratigraphischer Hinsicht ist besonders das Profil von Zamarovce wichtig, welches Fr. ProšEK \& V. LOžEK (1955) beschrieben haben. Dieses Profil entspricht in allen Hinsichten unseren Erkenntnissen; der dritte begrabene Boden wäre unser interglazialer Bodenkomplex, der zweite Boden unser W 1/2. Der Möglichkeit, daß es sich um ein älteres Interstadial handeln könnte, widersprechen die Funde des Szeletien. Auch die aus der Molluskenfauna abgeleiteten Klimabedingungen entsprechen unseren Feststellungen an der Säugetiergemeinschaft. Besonders gut ist das Auftreten der kälteliebenden Fauna im W 2 im Gegensatz zu den früheren Gemeinschaften zu sehen.

Aus den Arbeiten von LožEK geht hervor, daß sich die interglaziale Malakofauna von der interstadialen deutlich unterscheidet. Beachtenswert ist aber die Lage der interglazialen Molluskenfunde, die meist im obersten Teile des Lösses unterhalb des Bodenkomplexes vorkommen (Letky, Jenerálka, Nové Město n/Váh., Dolní Věstonice, Zamarovce). V. LOŽEK nimmt an, daß es sich in diesen Fällen um verlagerte Schneckenschalen handelt (Klíma, Kukla, LožEK, DE VRies 1962); mit Rücksicht darauf, daß diese Erscheinungen an vielen Lokalitäten vorkommt und ständig sich wiederholt, vermuten wir, daß diese Tatsache durch andere Umstände verursacht ist.

Auch vom a rchäologischen $\mathrm{S}$ tandpunkte kann man zur Würmgliederung einen grundsätzlichen Beitrag bieten. In ganz Europa erscheinen die Kulturen des Jungpaläolithikums ungefähr zur gleichen Zeit, was an vielen Stationen in West-, Mittelund Osteuropa nachgewiesen wurde, und zwar in einem bestimmten Abschnitt des Mittelwürms. Die frühesten jungpaläolithischen Industrien werden in Böden gefunden, die durch Radiocarbondaten etwa zwischen 32.000 und 40.000 datiert werden. Von einer Reihe Freiland- und Höhlenstationen erwähnen wir z. B. Zamarovce und Ivanovce (mit Szeletien), die Höhle Čertova pec (Szeletien), Vlčkovce (Szeletien), die Höhle Pod hradem (Szeletien, Olschewien). Das Erscheinen des Jungpaläolithikums ist auf diesen Lokalitäten mit der Zeit der mittelwürmzeitlichen Bodenbildung (W 1/2) verknüpft und bildet so einen Leithorizont, den man mit anderen Perioden nicht verwechseln kann. 


\section{Auswertung}

Eine der wichtigsten Fragen der Würm-Stratigraphie bleibt die Frage der Existenz des Mittelwürm-Interstadials (W 1/2). In dieses Interstadial verlegte man früher den Boden von Göttweig, mit dessen Namen man später auch diese Warmschwankung benannte. Als auf Grund weiterer Erkenntnisse klar wurde, daß der Göttweiger Boden in einer älteren Warmzeit gebildet worden war, wirkte es sich so aus, daß ihre Existenz auch in Abrede gestellt wurde. Demgegenüber nehmen wir an, daß der Boden von Göttweig irrtümlich in dieses Mittelwürm-Interstadial verlegt wurde, und daß seine richtige Einreihung kein genügender Grund für die Ablehnung dieses Interstadials sein kann.

Der Boden dieser Periode befand sich in Modřice, wie wir oben gezeigt haben, hoch über dem Waldboden, der von allen Teilnehmern der Kommission für Lößstratigraphie der INQUA im Sommer 1963 übereinstimmend als die Bodenbildung des letzten Interglazials (PK III) bezeichnet worden ist. Eine ganz analoge Situation konnten wir auch an anderen Löß- und Höhlenaufschlüssen beobachten. Dieser mittelwürmzeitliche Boden unterscheidet sich vom letzten Interglazial nicht nur durch seine stratigraphische Lage, sondern auch durch die eingeschlossenen paläontologischen und archäologischen Funde.

Auch wenn man die bisherigen Radiocarbondaten nur mit Vorbehalt berücksichtigt, muß man doch feststellen, daß es bereits eine ganze Reihe von Daten gibt, die dieses Interstadial zeitlich gut begrenzen und eine große Zeitspanne zwischen ihm und dem Interglazial nachweisen. Zu den bekannten Daten (vgl. Movius 1960) führen wir fol-

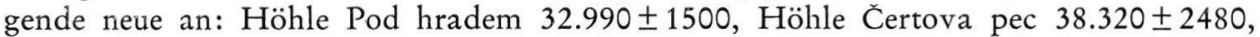

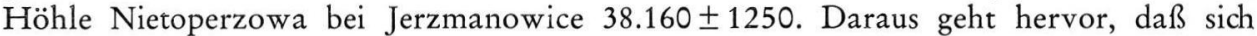
diese Schwankung vom letzten Interglazial und auch von den Frühwürm-Interstadialen gut unterscheidet.

Relativ nahe in der absoluten Chronologie steht aber diese Schwankung dem letzten schwachen Interstadial (W 2/3), so daß die Vermutung entstehen könnte, es handle sich um eine und dieselbe Periode. Das ist aber infolge der paläontologischen und archäologischen Funde unmöglich. An allen bekannten Lokalitäten zeigen die beiden gemeinsam auftretenden Fundbestände einen starken Unterschied. Die Faunengemeinschaft des Mittelwürminterstadials hat eine ganz andere Prägung als die kalte Fauna des W 2/3; die für das W 2/3 typische hochentwickelte Kultur des Ostgravettien kann man nicht mit den altertümlichen Industrien des Szeletien und Aurignacien verwechseln. Dieses hier betrachtete Interstadial liegt also chronologisch zwischen den altwürmzeitlichen Schwankungen (Amersfoort, Brörup) und der schwachen Verlehmung des W 2/3.

Bisher haben wir in unseren Arbeiten die einzelnen Würmstadiale und Interstadiale mit der Abkürzung W und einer Nummer bezeichnet. Mit Rücksicht darauf, daß durch neue Erkenntnisse die Anzahl der Würmschwankungen vergrößert wurde, entspricht diese Bezeichnungsweise nicht mehr dem wirklichen Stand. Deshalb neigen wir zur Benennung der Interstadiale mit Namen typischer Lokalitäten, wobei wir uns der Schwierigkeiten bewußt sind, welche bei der Bezeichnung der einzelnen Stadiale entstehen werden.

Die blasse Verlehmung W 2/3 wurde schon früher nach der Lokalität Stillfried als Stillfried B bezeichnet. Mit dieser Periode deckt sich unserer Meinung nach nicht die Bezeichnung „Paudorf“, von dem wir auf Grund seiner Bodenentwicklung und der Parallelisierung mit anderen Aufschlüssen vermuten, daß es sich um ein älteres Interstadial handelt, höchstwahrscheinlich um das hier behandelte W 1/2. Die Bodenbildung W 1/2 wurde früher als Göttweig bezeichnet. Es stellte sich aber heraus, daß der Boden an dieser klassischen Lokalität dem letzten Interglazial angehört, so daß die Mittelwürmschwankung ohne Benennung bleibt. Deshalb schlagen wir vor, dieses Interstadial als In t e r stadia l Podh rade m zu bezeichnen, weil sein Boden an dieser Lokalität gut ausgeprägt ist, in klarer stratigraphischer Position liegt, durch $\mathrm{C}^{14}$ datiert und paläontologisch wie auch archäologisch belegt ist. 
Ein wichtiger stratigraphischer Horizont in unseren Lössen ist der Komplex mächtiger eemzeitlicher Böden. In optimalen Fällen ist er ziemlich gegliedert. Bei unseren früheren Arbeiten haben wir in seinem oberen Drittel eine durch typischen Löß gebildete Schicht festgestellt. Diesen Löß haben wir als Präwürm und den ganzen Komplex zusammen als Riß/Würm bezeichnet. Auf Grund neuer Forschungen reihen wir ins R/W bloß den unteren Teil dieses Komplexes ein, und zwar im Liegenden des Lösses, während sein oberer Teil schon dem Amersfoort-Interstadial entsprechen dürfte. Diesen ganzen Bodenkomplex bedeckt eine Lößschicht, in deren Hangenden in optimalen Fällen noch eine Verlehmungszone liegt, die stratigraphisch dem Brörup entspricht. Für die Benennung der einzelnen Würmstadiale könnte man dann diese Bezeichnungen benützen: Frühwürm, Altwürm, Mittelwürm, Jungwürm.

Wir halten es für nötig, weiter noch auf geringe Oszillationen aufmerksam zu machen, die wir mehrmals und in verschiedener Anzahl im Löß zwischen dem R/W-Komplex, resp. dem Brörup und dem folgenden Interstadial Podhradem beobachtet haben. Es handelt sich immer um blaßbraune Zonen, in der Mächtigkeit von etwa $30 \mathrm{~cm}$, bei deren Bildung es wahrscheinlich nicht zu einer Unterbrechung der Lößanwehung kam, sondern nur zu einem bedeutend geringeren Nachschub des Staubes, was eine schwache Verlehmung ermöglichte. Meist findet man nur e in e solche Verlehmung; in optimalen Fällen haben wir aber auch drei ähnlich ausgebildete und durch typischen Löß getrennte Zonen gefunden (Ziegelei am Roten Berg in Brno.). Offenbar handelt es sich nicht nur um eine lokale Erscheinung; eine solche Zone befindet sich auch in den klassischen Aufschlüssen in Göttweig und Paudorf zwischen dem „Göttweiger" und „Paudorfer" Boden.

Schon in den früheren Arbeiten haben wir auf die auffallende Ubereinstimmung unserer diskutierten Lößprofile mit den Travertinaufschlüssen bei Ehringsdorf aufmerksam gemacht. Auch heute vertreten wir dieselbe Meinung, nur die stratigraphische Interpretation nähern wir besser den neuen Erkenntnissen an. Die unteren Travertine von Ehringsdorf mit ausgesprochen interglazialer Fauna würden in dieser Arbeitshypothese dem unteren Bodenkomplex in unseren Lössen, das ist dem Eem, entsprechen. Die Schicht des Parisers bedeutet, obzwar es sich nicht um einen richtigen Löß handelt, eine Ablagerung einer kühleren, nicht kalten, mehr kontinentalen Klimaphase (GUENTHER 1958). Nach pollenanalytischen Untersuchungen von $\mathrm{H}$. ЈАсов herrschte während der Bildung des Parisers eine gemäßigte Kälte (in Beнm-Blancke 1960). Die Vertebratenfunde zeugen davon, daß die Grenze zwischen dem unteren Travertin und dem Pariser eine bedeutende Veränderung in der ganzen Gemeinschaft infolge veränderter Lebensbedingungen bedeutet. Die Fauna aus dem Pariser nähert sich durch ihre Zusammensetzung eher jenen aus dem oberen Travertin. Dabei handelt es sich nicht um eine ausgesprochen kalte Fauna, aber um eine Gemeinschaft, deren Arten (Kahlke 1958 - Ehringsdorf, oberer Travertin I) z. B. der Fauna unseres Interstadials Podhradem entsprechen. Man muß sich bewußt bleiben, daß die ersten Spuren einer ausgeprägten kalten Fauna erst nach diesem Interstadial Podhradem erscheinen, und daß die früheren Würm-Interstadiale vermutlich eine einander ähnliche Gemeinschaft aufweisen werden. Daher ist es nicht ausgeschlossen, daß der Pariser einen gewissen zeitlichen Hiatus in sich verbirgt, dem auch das plötzliche Erscheinen einer neuen Faunengemeinschaft entsprechen würde, wobei der verflossene Boden auf seiner Oberfläche und der hangende obere Travertin I das Produkt des Amersfoort-Interstadials sein könnten. Die Lage des Parisers könnte man dann mit der Lößschicht im Bodenkomplex aus unseren Lößaufschlüssen vergleichen.

Säugetierfunde aus dem oberen Travertin II sind nach H.-D. KAHLKE ein wenig verschieden von jenen des oberen Travertins I. Beim Versuch einer Parallelisierung mit unseren Profilen könnte man annehmen - natürlich erst hypothetisch, ohne es beweisen zu können - daß man diesen oberen Travertin II der braunen verlehmten Zone über dem Bodenkomplex (Eem + Amersfoort), die wir dem Brörup eingliedern, gleichsetzen kann. 
Der Pseudopariser, dessen Fauna nach Kahlke Anklänge an die des Parisers zeigt, wäre dann zeitlich mit unserer Lößschicht $\mathrm{zw}$ ischen dem Amersfoort und Brörup identisch.

Diese stratigraphische Eingliederung der Travertine von Ehringsdorf unterstützt auch das Deckprofil, welches nach E. W. Guenther (1958) in kompletter Ausbildung die Schichten des Mittel- und Jungwürms enthält. Den fossilen Bodenhorizont (verlagerter Böden) setzen wir dem Interstadial Podhradem gleich.

\section{Ergebnisse}

Eine ganze Reihe von Arbeiten aus den vergangenen Jahren zeigt, daß die Anzahl der in den Lößprofilen festgehaltenen Oszillationen aus der Zeit des Würms viel größer ist, als man früher dachte. Der mächtige durch einen Löß gegliederte Bodenkomplex, den wir in unseren früheren Arbeiten dem letzten Interglazial gleichsetzten, umfaßt auch die Frühwürm-Interstadiale. Zum Eem gehört nur der untere Teil dieses Komplexes, und die erwähnte Lößschicht zeigt schon den Antritt der beginnenden Schwankungen der letzten Eiszeit an. Der restliche obere Teil des Bodenkomplexes gehört schon dem Amersfoort-Interstadial und die unmittelbar folgende, durch einen weiteren Löß abgetrennte Verlehmungszone dann dem Brörup-Interstadial an. Den Boden des Interstadials Podhradem trennt gewöhnlich von den vorangehenden ein mächtiger Löß, so daß er bei optimaler Ausbildung der Würmsedimente dem Endwürm näher steht als der tieferliegenden Bodenschichtenfolge. Das Interstadial Stillfried B ist immer nur als eine schwache Verlehmung entwickelt; es dürfte sich also wahrscheinlich nur um eine kurze Oszillation handeln, die in ihrer Intensität sehr ähnlich jenen schwachen Oszillationen ist, die wir im Altwürmlöß zwischen Brörup und Podhradem gefunden haben.

\section{Schriftenverzeichnis}

Benm-Blancke, G.: Altsteinzeitliche Rastplätze im Travertingebiet von Taubach, Weimar, Ehringsdorf. Alt-Thüringen 4, Weimar 1960.

FINK, J.: Protokoll über die vom 22.-28. 8. 1963 in der ČSSR stattgefundene Tagung mit Exkursionen. INQUA-Subkommission für Lößstratigraphie, Wien 1963. - Vgl. auch Bericht in Eiszeitalt. u. Gegenw. 15, 229-235, 1964.

Guenther, E. W.: Feinstratigraphische Untersuchung einer Zwischenschicht und der Deckschichten der Travertine von Ehringsdorf. Alt-Thüringen 3, 1-15, Weimar 1958.

KAhlKe, H. D.: Die jungpleistozänen Säugetierfaunen aus dem Travertingebiet von TaubarWeimar-Ehringsdorf. Alt-Thüringen 3, 97-130, Weimar 1958.

KLíma, B.: Die archäologische Erforschung der Höhle Švédův stůl in Mähren. Anthropos, N. S. 5, 7-96, Brno 1962.

Klíma, B., Kukla, J., LožEK, V. \& de VRies, Hl.: Stratigraphie des Pleistozäns und Alter des paläolithischen Rastplatzes in der Ziegelei von Dolní Věstonice (Unterwisternitz). Anthropozoikum 11 (1961), 93-145, Praha 1962.

LožEK, V.: Měkkýšsi českolovenského kvartéru. (Mollusken des tschechoslowakischen Quartärs.) Rozpr. úst. geol. 17, Praha 1955. - - Zasedání Subkomise INQUA pro stratigrafii spraší v Československu. Věstn. UUUG 34/3, 233-237, Praha 1964.

LožEK, V. \& KukLA, J.: Das Lößprofil von Leitmeritz an der Elbe, Nordböhmen. Eiszeitalter u. Gegenwart 10, 81-104, Ớhringen 1959.

Movius, H. L.: Radiocarbon dates and Upper Palaeolithic archaeology in Central and Western Europe. Current Anthropology I/5-6, 355-391, Chicago 1960.

Musıl, R.: Die Bärenhöhle Pod hradem. Die Entwicklung der Höhlenbären im letzten Glazial. Anthropos, N. S. 10, 9-22; Brno 1965.

Musil, R., Valoch, K. \& Nečesaný, Vl.: Pleistocenní sedimenty okolí Brna (The pleistocene sediments in the vicinity of Brno). Anthropozoikum 4 (1954), 107-167, Praha 1955.

Pelíšek, J.: Př́íspěvek ke stratigrafii spraší svrateckého úvalu. (A contribution to the strat:graphy of loess in the Svratka river valley, Czechoslovalkia.) Acta Acad. Sci. Nat. Mor. Sil., 21/11, 1-19, Brno 1949.

ZAGWIJN, W. H.: Vegetation, climate, and radiocarbon datings in the Late Pleistocene of the Netherlands. Meded. Geol. Sticht. N. S. 14, 15-45, Maastricht 1961.

Manuskr. eingeg. 16. 12. 1965.

Anschrift der Verf.: Dr. R. Musil und Dr. K. Valoch, Brno, ČSSR, Moravské Museum Ustav Antropos. 